\title{
Envisioning Reflective and Relaxing Design with ASMR
}

Josephine 'Jolie' Klefeker ATLAS Institute

University of Colorado Boulder Boulder, CO, 80305, USA

Josephine.klefeker@colorado.edu

\section{Laura Devendorf}

ATLAS Institute \& Dept. of

Information Science

University of Colorado Boulder Laura.devendorf@colorado.edu

Permission to make digital or hard copies of part or all of this work for personal or classroom use is granted without fee provided that copies are not made or distributed for profit or commercial advantage and that copies bear this notice and the full citation on the first page. Copyrights for thirdparty components of this work must be honored. For all other uses, contact the Owner/Author.

DIS '19 Companion, June 23-28, 2019, San Diego, CA, USA (C) 2019 Copyright is held by the owner/author(s)

ACM ISBN 978-1-4503-6270-2/19/06.

https://doi.org/10.1145/3301019.3323903

\begin{abstract}
In this paper, we aim to acquaint the sensory phenomenon of Autonomous Sensory Meridian Response (ASMR) with the DIS community and investigate its relevance via research through design. We inquire if the aesthetics of ASMR media can inspire the design of technology that is reflective and relaxing. In order to do so, we created a sonic toolkit that encourages users to explore their environments and make recordings inspired by the detail-oriented, intimate, and mundane nature of ASMR media. Over the course of a self-directed user study, we invited five participants to engage the toolkit and interviewed them about their experience. We present a description of ASMR and its connection to ongoing conversations within DIS as well as our findings from the user study. We conclude with a provocation which suggests that fostering relaxing designs may be as simple as providing people with a microphone.
\end{abstract}

\section{Author Keywords}

ASMR; Reflective Design; Research through Design; Binaural Audio; Slow Technology.

\section{CSS Concepts}

- Human-centered computing Interface design prototyping 

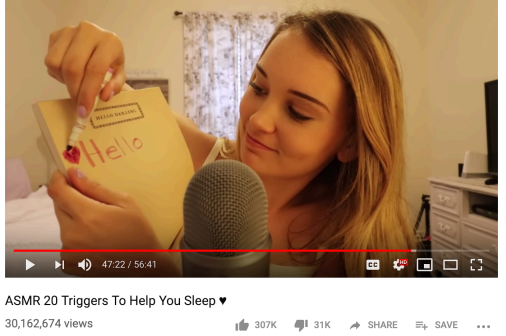

Figure 1: ASMR Darling uses drawing to "trigger" her viewers

Figure 2: ASMR Design Characteristics

\section{1) Generic Personalization:}

Creators carefully use language to feign a personal relationship with viewers. For example, they may talk to the camera as if they are talking to an individual despite the fact that an audience is watching. This strategically simulates a personal appeal without the effort of customization.

2) Familiarity: ASMR often utilizes familiar and mundane objects and situations for relaxation. By relying on situations that viewers are most likely already comfortable with, the content becomes relatable which helps viewers to connect the relaxing tone of the video with their own associations [1], potentially creating a more powerful experience.

\section{Introduction}

ASMR can be described as a relaxing, tingling sensation, usually in the head and neck, in response to certain sensory stimuli. Some suggest that ASMR is euphoric, or even capable of alleviating anxiety, depression, insomnia, and stress $[3,4]$. An individual's ASMR or preference to a certain sound is colloquially referred to as a "trigger." Triggers can be sorted into several popular categories such as tapping, scratching, whispering, or slow movements. While audiovisual stimuli are most common, some say ASMR can be elicited by any number of auditory, visual, tactile, or olfactory sensations [5]. What causes ASMR media's aesthetics and practices to be beneficial, and the degree to which that aesthetic maps onto interactive products (such as wearables, objects, etc.) remains unknown. As such, our design inquiry is motivated by asking: In what way might the aesthetics and narratives of ASMR media inform the design of interactive systems? Specifically, could they help designers create systems that are beneficial (i.e. reflective and relaxing) in similar ways to ASMR?

The digital communities that have emerged around ASMR are a fascinating microcosm, centered around relaxation, sleep, and "tingles." With over 30 million views, ASMR Darling's "ASMR 20 Triggers To Help You Sleep $\bullet "$ " [2] is one of the most viewed ASMR videos of all time and a prime example of popular, ASMR media (Figure 1). "This is all for you..." she whispers, as she gestures toward the camera lens, as if she's fussing with your hair. The triggers in this compilation range from tapping on a book to a roleplay haircut with a variety of sounds in between. While most of these triggers are seemingly random, pointless, and odd, ASMR Darling seems unfazed as she slowly, deliberately, and carefully makes her way through the miscellanea before her. Featuring these idiosyncratic, unfamiliar cultural and aesthetic paradigms, we believe that ASMR media contains untapped inspiration for designers and novel ways of conceptualizing humancomputer relationships. Inspired by slow technology [7] and research through design [6], we inquire if ASMR can provide a new way of designing technology that is reflective [8] and relaxing.

In this provocation, we analyze the aesthetic choices made by ASMR creators and inquire about the ASMR experience outside of YouTube. To learn more about ASMR in the physical world, we interviewed the creator of WhisperLodge, an ASMR-theatre performance that offers in-person, ASMR experiences. Next, we created a prototype that incorporated characteristics we found in ASMR media. This took the form of a sonic toolkit that encourages users to explore their environments and make recordings inspired by the detail-oriented, intimate, and mundane nature of ASMR media. We present a brief summary of our findings to show how participants focused almost entirely on the binaural recording equipment the kit provided. We conclude with a provocation that suggests that fostering the kinds of experiences we envision might not require radical innovation: maybe it is as simple as providing people with a microphone.

\section{Design Process}

Our engagement with ASMR began with an in-depth inquiry into its aesthetics and practices. To better understand its relevance to design, we analyzed the successful features of ASMR media and attempted to distill those aesthetic qualities into distinct characteristics that we can apply to the design of 
3) Gestures of Closeness: ASMR media often repurposes actions associated with physical closeness to evoke a sense of proximity. Such actions are often used to supplement an ASMR scenario and would usually not be appropriate in reality. For example, whispering in a patient's ear during a medical roleplay or caressing a client's face during a haircut.

\section{4) Personal Attention and} Interest: ASMR creators pay special attention to their audience members and their wellbeing, taking significant time to inquire about their audience's lives. For example, asking the audience

"how they are" and emphasizing that the content is designed with their relaxation in mind. This fosters a sense of care and trust between creator and audience.

5) Digital Distance: Because it's hosted on platforms like YouTube and Instagram, ASMR media is accessible, convenient and able to be viewed privately. The existence of several subcommunities helps users to tailor their experience. This also enables ASMR content to be much slower than the real-life situations by which it is inspired.

\section{6) Subdued Light and Color:}

Color palettes and brightness are frequently muted and subdued to cultivate a more relaxing environment. technologies. We chose to focus on what the community calls, "intentional ASMR," media made specifically to provoke ASMR sensations. Because intentional ASMR is "designed" to provoke tingles, we found its unique characteristics to be more visible. We randomly chose videos from five of the most subscribed ASMR channels on YouTube and noted as many salient characteristics as possible. From this analysis, we grouped common themes and found that most videos exhibited several distinct characteristics, which are outlined in the sidebar in Figure 2.

To discuss how we might translate the aesthetics and narratives of video content into physical, everyday spaces, we conducted an interview with Melinda Lauw, co-founder of WhisperLodge. WhisperLodge is a live immersive theatre performance focused on ASMR. Their performances are all in-person, either in small groups or one-on-one. Lauw offered her insight into the key differences between the online and in-person ASMR experience as, "the vulnerability that the person has to be open to...they've got to be open to putting themselves next to someone else." Lauw's interview made us aware of the potential for powerful, emotional in-person ASMR experiences. Additionally, it helped us feel that applying ASMR to design would be possible, but also suggested that users may feel strange and vulnerable while engaging these systems.

\section{Design Concept}

To explore the relationship between ASMR and design, we developed a sonic toolkit that invited users to explore sounds produced by mundane objects and interactions. The toolkit functioned as a reflective design or probe $[6,8]$, helping us inquire into the relationships between reflection, relaxation, and playful sonic engagements. The kit was influenced by the design characteristics in Figure 2 and consisted of a binaural recording kit, finger covers, and a set of written prompts for action. Please note that the specific characteristics that informed each element of the kit are indicated in parentheses.

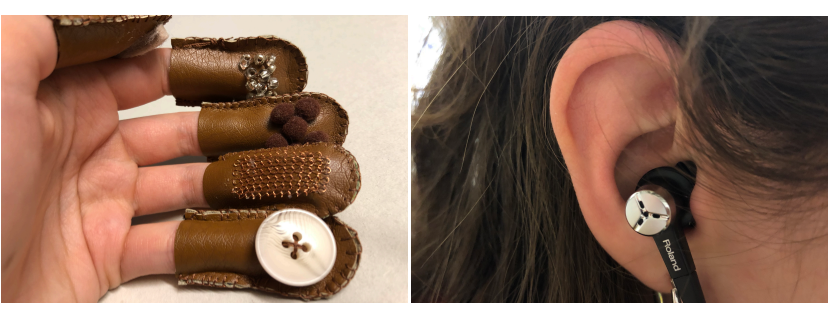

Figure 3: Finger covers and binaural headphone/microphones.

The kit's audio recording equipment was modeled after a common setup for current ASMR creators. We chose a set of Roland binaural recording headphones, seen in Figure 3. Binaural audio, already common in ASMR videos, is a form of capture where two microphones are placed in the same position as one's ears to provide a realistic, 3D audio playback. It gives way to an intense, almost surreal exaggeration of surrounding noise which we implemented as an attempt to foster an attention to detail in participants $(2,5)$. The finger covers (Figure 3 , left) were an attempt to encourage active engagement with sound. Inspired by the tapping and probing of ASMR creators (3), each finger was designed with materials that elicit unexpected sounds $(1,6)$. The prompts were an opportunity to incorporate generic personalization (1) and familiarity (2) into the kit. We wanted the prompts to evoke a feeling of care and comfort and invite users to interact with their 

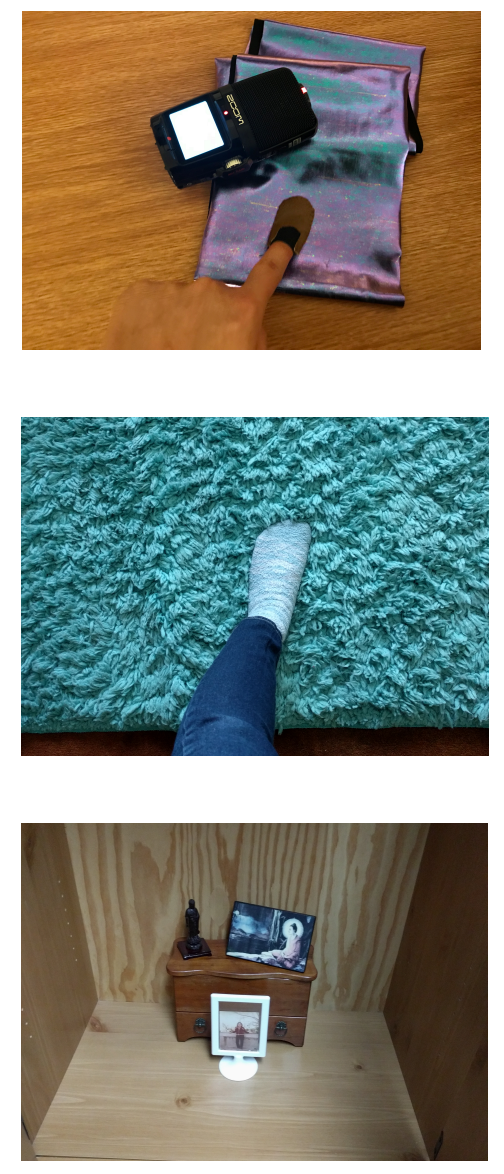

Figure 4: Participant photos illustrating their recordings. The topmost image is a participant using a finger cover, the middle is illustrative of a mindfulness meditation, and the bottom is a participant's altar which is a part of their spiritual practice. surroundings in ways they may otherwise consider to be strange (4).

\section{Methods}

We asked five participants to carry the kit with them for one day and then interviewed them about their experience. We recruited participants through university email lists focused on art and design. Participants had diverse levels of experience with design, audio recording and ASMR. Participants were not informed of the toolkit's connection to ASMR prior to the study. We asked them to make at least three separate recordings over the course of their day as well as take illustrative photos of each recording on their smartphones. Participants were not required to use the prompts or finger covers, they were offered as inspiration to them rather than mandatory.

\section{Findings and Discussion}

Upon completing the study, interviews suggested that participants found the experience to be enjoyable and noted a fresh perspective, heightened awareness, or a newfound appreciation for the sounds in their familiar surroundings. Specifically, participants described the experience of recording with the kit as "mindful" and "feeling in the moment." Two of them also explained the experience as providing newfound appreciation for "background noise." Each participant used the kit in a distinctive way, some preferred listening through the binaural headphones in real time while others prioritized replaying the recordings. A wide variety of environments were engaged as well: offices, bedrooms, friend's houses, cars, etc. (Figure 4). However, they all steered away from unfamiliar, public spaces.

Additionally, participants recorded a vast array of sounds from a 45-minute-long family dinner to their hands in the stream of an air vent.

Each participant offered a distinctive viewpoint and insight, but interviews ultimately suggested promise as well as limitations. The participants experienced what they described as "mindfulness" through the

heightened awareness brought on by recording. Yet, the finger covers and prompts were barely used. We think this is because they found plenty of inspiration in recording alone and/or the finger covers were not always readily accessible. From these early results, we are still confident that ASMR media is capable of informing relaxing and reflective design in meaningful ways, but we feel that more information is required to support that claim. In continuing this research, we plan to apply our ASMR characteristics and findings to the design of an interactive wearable system.

\section{A Provocation}

Our most salient finding from this study was the high interest in recording but under-use of the prompts and finger covers. We believe that the primary benefit of our kit was that it simply gave people permission to engage this audio equipment, who wouldn't have sought it out otherwise. While DIS is a community largely focused on developing novel systems and technologies, our study suggests that supporting relaxing interactions with technology may be as simple as giving people a high-quality microphone and in doing so, giving them permission to engage with their everyday environment in a fresh and unexpected way. 


\section{References}

[1] Andersen, J. 2015. Now You've Got the Shiveries: Affect, Intimacy, and the ASMR Whisper Community.

Television \& New Media. 16, 8 (Dec. 2015), 683-700. DOI:https://doi.org/10.1177/1527476414556184.

[2] ASMR Darling ASMR 20 Triggers To Help You Sleep v. https://www.youtube.com/watch?v=DaXwnTkOhUE.

[3] Barratt, E.L. and Davis, N.J. 2015. Autonomous Sensory Meridian Response (ASMR): a flow-like mental state. PeerJ. 3, (Mar. 2015), e851.

DOI:https://doi.org/10.7717/peerj.851.

[4] del Campo, M.A. and Kehle, T.J. 2016. Autonomous sensory meridian response (ASMR) and frisson:

Mindfully induced sensory phenomena that promote

happiness. International Journal of School \&

Educational Psychology. 4, 2 (Apr. 2016), 99-105.

DOI:https://doi.org/10.1080/21683603.2016.1130582.

[5] Fredborg, B.K. et al. 2018. Mindfulness and autonomous sensory meridian response (ASMR). PeerJ. 6, (2018), e5414.

DOI:https://doi.org/10.7717/peerj.5414.

[6] Gaver, W. 2012. What should we expect from research through design? Proceedings of the 2012 ACM annual conference on Human Factors in Computing Systems - CHI '12 (Austin, Texas, USA, 2012), 937.

[7] Hallnäs, L. and Redström, J. 2001. Slow Technology - Designing for Reflection. Personal Ubiquitous Comput.
5, 3 (Jan. 2001), 201-212.

DOI:https://doi.org/10.1007/PL00000019.

[8] Sengers, P. et al. 2005. Reflective Design. Proceedings of the 4th Decennial Conference on Critical Computing: Between Sense and Sensibility (New York, NY, USA, 2005), 49-58. 